\title{
Philonsorbonne
}

$7 \mid 2013$

Année 2012-2013

\section{Doctorales 2012}

Résumés des interventions des secondes Doctorales de Philosophie (2012)

\section{(2) OpenEdition}

\section{Journals}

Édition électronique

URL : https://journals.openedition.org/philonsorbonne/521

DOI : 10.4000/philonsorbonne.521

ISSN : 2270-7336

Éditeur

Publications de la Sorbonne

\section{Édition imprimée}

Date de publication : 15 novembre 2013

Pagination : 121-145

ISSN : 1255-183X

Référence électronique

«Doctorales 2012 », Philonsorbonne [En ligne], 7 | 2013, mis en ligne le 18 décembre 2013, consulté le 09 juin 2021. URL : http://journals.openedition.org/philonsorbonne/521 ; DOI : https://doi.org/

10.4000/philonsorbonne.521 


\section{DOCTORALES 2012 ${ }^{(*)}$}

Matthieu AMAT

Laboratoire de rattachement : PhiCo/EXeCO

Thèse dirigée par : Philippe Büttgen

matthieuamat@gmail.com

\section{Esprit objectif, esprit objectivé et objectivations dans la philosophie de la culture de Georg Simmel}

La seconde section du sixième chapitre de la Philosophie de l'argent de Simmel (1900) est consacrée à «la relation discordante entre culture objective et subjective», entre l'ensemble des produits du «travail spirituel» de l'humanité - sciences, arts, technique, etc. - et la culture des individus. La quantité de culture objective crôit exponentiellement tandis que la culture des individus stagne ou régresse. Concluant presque l'ouvrage, la réflexion sur ce problème apparaît à Simmel comme un moyen particulièrement fécond pour l'intelligence de la modernité. Il ne cessera d'ailleurs d'y revenir, depuis Les grandes villes et la vie de l'esprit (1903) jusqu'à La crise de la culture (1918).

Le problème a souvent été abordé à partir de la tension entre la vie et les formes. Cette perspective s'autorise des derniers textes de Simmel, du Simmel «philosophe de la vie». Si elle est tout à fait légitime, elle peut toutefois faire oublier que Simmel est aussi philosophe de l'esprit, qu'il ne situe pas le mouvement du seul côté de la vie mais, sans en faire un sujet, envisage des lois et des processus immanents à l'esprit, qu'il nomme alors esprit objectif. Nous avons voulu montrer que la philosophie de la culture de Simmel est indissociable d'une philosophie de l'esprit et notamment d'une réflexion sur les différents modes d'objectivation de l'esprit.

(*). Sont ici publiés les résumés des différentes interventions ayant eu lieu lors des Doctorales de Philosophie organisées à la Sorbonne, les $1^{\text {er }}$ et 2 juin 2012, par les doctorants Florent FranchetTe (IHPST), Refik GürEMEN (Gramata), Baptiste MonsaingeOn (CETCOPRA), Elise Sultan (CHSPM) et Alexandre TANASE (PhiCo). 
Comprendre les mouvements propres à la culture objective nécessite en effet de la penser comme de l'esprit objectivé. Celui-ci est objectivation (Vergegenständlichung) de la vie, effet de son travail. Mais cela ne suffit pas à le décrire : d'un autre point de vue l'esprit objectivé est actualisation de l'esprit objectif, entendu comme un «royaume idéal des valeurs théoriques ». Nous avons voulu mettre en avant la façon originale dont Simmel s'approprie en ce point la théorie platonicienne des idées. L'étrangeté et l'autonomisation de la culture objective sont appréhendées à l'aide d'un modèle métaphysique et pas seulement à partir d'analyses socio-historiques. «La splendeur et la grandeur de la culture moderne, écrit Simmel, présentent ainsi quelque analogie avec ce lumineux royaume des idées chez Platon, où l'esprit objectif des choses est là dans sa perfection immaculée ».

Dans un deuxième temps, nous avons montré que cette tension de la culture moderne se reflétait dans la structure de la personnalité, soumise à un autre processus d'objectivation, la réification (Versachlichung). Nous avons toutefois montré que celle-ci ne saurait détruire le foyer de la subjectivité et la révélait plutôt : processus simultané de subjectivation. Cependant, les développements immanents à la culture objective réduisant constamment la valeur culturelle de ses contenus - leur valeur pour le développement de l'individu qui s'y rapporte -, cette subjectivité délivrée par la modernité menace de rester vide, formelle. Sur ces deux derniers points, nous nous sommes appuyés sur les Problèmes fondamentaux de la philosophie (1910) et sur Le concept et la tragédie de la culture (1911).

À la lumière de cette philosophie de l'esprit sont finalement apparues les limites des représentations dialectiques (la culture objective peut en dernier lieu être réappropriée par la vie subjective) ou expressivistes (la culture objective est extériorisation de la vie) en philosophie de la culture. 
Thibault BARRIER

Laboratoire de rattachement : CHSPM Thèse dirigée par : Chantal Jaquet

thbarrier@gmail.com

\section{«Que pour les yeux» Admiration et excès de puissance chez Corneille}

Si Corneille présente la plupart de ses textes théoriques comme des commentaires de la Poétique d'Aristote, il est un point sur lequel il prétend toutefois rompre avec cet héritage et affirmer son originalité : le statut de l'admiration.

L'Examen de Nicomède en fait la passion première, contre la crainte et la pitié, tout en conservant l'exigence cathartique. La « compassion » ne sert qu'à intéresser le spectateur au sort des personnages mais ne permet plus de l'émouvoir. C'est à l'admiration que revient d'assurer l'effet purgatif de la tragédie. Au lieu d'exciter la crainte et la pitié à la vue des malheurs auxquels conduit un vice, il est préférable de susciter l'admiration pour la vertu contraire. Cette substitution vise l'efficacité : l'admiration pour une vertu entraîne ipso facto une aversion pour le vice opposé, mais elle est moins risquée car elle épargne au spectateur le risque de contamination par le vice qui s'expose sur scène. La fonction de l'admiration est donc indexée sur la mise à distance à laquelle elle procède. Alors que la pitié attache l'esprit aux intérêts du personnage, l'admiration lui ménage une position de retrait.

Une fois ce geste repéré, reste à déterminer l'objet de l'admiration. Loin de faire de la vertu seule cet objet, le premier Discours refuse l'identification entre la «bonté » dramatique du personnage et sa vertu morale. "Vertueuse ou criminelle $»^{1}$, cette bonté se situe en deçà de la distinction entre bien et 
mal, et désigne plutôt la grandeur d'un caractère qui exhibe une puissance d'action ferme, soustraite à l'irrésolution et aux variations des circonstances. Le spectateur peut aimer ou haïr les actions selon qu'elles sont vertueuses ou vicieuses, mais il ne peut qu'admirer la puissance singulière et indifférenciée qui en est la source. L'admiration reconduit le regard au-delà du régime superficiel des approbations morales pour ne plus considérer que le pur exercice d'une puissance. La fermeté des résolutions de Cléopâtre ou la vivacité d'esprit de Dorante sont deux modalités d'une même "masse de $[. .$.$] puissance »^{2}$ qui excède les forces rivales, et constitue l'objet propre de l'admiration.

«Que pour les yeux » ${ }^{3}$ désigne à la fois l'esthétique du spectaculaire (plein les yeux) mais aussi une mise à distance qui interdit la fusion passionnelle du spectateur (seulement les yeux). L'admiration fait voir plus, car elle porte sur la source des actions, tout en maintenant un écart, dessinant ainsi la juste distance du spectateur à l'égard du spectacle.

2. Examen de Nicomède, in Euvres complètes, Paris, Gallimard, «Bibliothèque de la Pléiade », 1981-1987, t. II, p. 643. Cette expression précède immédiatement le passage sur l'admiration d'où nous sommes partis.

3. Formule par laquelle Corneille conclut l'Examen d'Andromède, in $O C$, éd. cit., t. II, p. 448. 
Filip BUYSE

Laboratoire de rattachement : CHSPM Thèse dirigée par : Chantal Jaquet filip.buyse1@telenet.be

\section{La physique de Spinoza : cartésienne dans les Principes et hobbesienne dans L'Éthique ?}

Le seul livre que Spinoza ait publié sous son nom de son vivant est une interprétation des Principia de Descartes. Un ouvrage qui avait commencé comme dictat d'un cours privé de la deuxième partie des Principia, partie que le philosophe français appelait souvent «Ma Physique». Il est clair que la physique de Spinoza dans cet ouvrage est cartésienne, bien qu'il y ait déjà des différences avec le texte de Descartes, souvent cachées, qui ont été commentées entre autre par Jonathan Israel ${ }^{4}$.

Dans la deuxième partie de l'Éthique, il y a aussi un traité physique entre les propositions 13 et 14. Cet Abrégé de physique n'est pas encore dans le Court Traité qui peut être considéré comme une proto-Éthique. Spinoza l'a donc ajouté pendant la période de rédaction de son chef d'œuvre. Étonnamment les deux physiques «spinozistes» sont différentes en ce qui concerne la formulation et le contenu.

Pourquoi Spinoza a-t-il changé ? Spinoza a probablement changé sous l'influence de De Corpore (1655) de Thomas Hobbes. Cet ouvrage avait été republié en 1668 dans une version adaptée par Johannes Blaeu à Amsterdam comme le premier tome de l'Opera Philosophica de Hobbes. En outre, Spinoza s'intéressait pendant cette période de rédaction de l'Éthique à la philosophie de «Hobbes ». Une année plut tôt, un de ses amis, Abraham van Berkel, avait traduit le Leviathan en néerlandais. L'œuvre de Hobbes 
était donc discutée dans le cercle de Spinoza. Curley ${ }^{5}$ a déjà remarqué l'influence du Léviathan sur le Traité théologico-politique (1670). Néanmoins l'influence de De Corpore est un point examiné dans la littérature secondaire.

Par exemple, une analyse de ce que Spinoza a écrit par rapport au «mouvement et repos » dans le lemme de la EIIp13 et de ce que Hobbes a écrit dans l'article 19 du chapitre XVIII de De Corpore permet de démontrer le rapport. Ce que Spinoza a écrit est très similaire à ce que Hobbes a écrit, mais très différent de ce que Spinoza avait écrit dans son interprétation de la première loi de la nature de Descartes. 
Marie DARRASON

Laboratoire de rattachement : IHPST

Thèse dirigée par : Jean Gayon

marie.darrason@gmail.com

\section{Pour une interprétation mécaniste de l'extension du concept de maladie génétique}

Le concept de maladie génétique a considérablement évolué depuis son origine tant en extension qu'en compréhension. Alors qu'il a d'abord été utilisé pour désigner des maladies monogéniques, mendéliennes et héréditaires, il s'applique maintenant à des maladies polygéniques, non mendéliennes et acquises à tel point qu'on a pu affirmer dans la littérature biomédicale contemporaine que toute maladie pouvait être considérée comme génétique 6 .

Cette affirmation a généralement été interprétée comme une tentative de génocentrisme, qui consacrerait la prédominance du rôle des gènes dans l'explication causale de la maladie au détriment des facteurs non génétiques. Comme il a été démontré que le génocentrisme est à la fois scientifiquement injustifié et éthiquement discutable ${ }^{7,8}$, cette affirmation devrait être rejetée. Il nous semble pourtant qu'à condition de bien vouloir cesser de mesurer l'influence causale des gènes et de l'environnement dans l'explication des maladies, c'est-à-dire à condition de sortir du problème de la sélection causale, il est possible de donner une interprétation pertinente de cette affirmation.

6. M.J. Khoury, «Genetics and genomics in practice : the continuum from genetic disease to genetic information in health and disease », Genetics in Medicine, 2003 : 4(5), p. 261-268.

7. D. Magnus, «The concept of genetic disease», in Health, disease and illness, Arthur L. Caplan, Jennifer J. McCarthney and Dominic A. Sisti (eds), Washington D.C, Georgetown University Press, 2004, p. 233-242.

8. K.C. Smith, «Towards an adequate account of genetic disease », in Establishing Medical realities, Harold Kincaid \& Jenifer M. McKitrick (eds), Dordrecht, Springer, 2007, p. 83-100. 
Nous proposons en particulier de nous appuyer sur la théorie génétique des maladies infectieuses ${ }^{9}$ qui prétend unifier les maladies infectieuses en mettant au jour quatre mécanismes génétiques communs à cette classe de maladies (prédisposition mendélienne à plusieurs infections, prédisposition mendélienne à une infection, gène majeur ou résistance à une infection, prédisposition polygénique). À partir de cet exemple d'une théorie génétique mécaniste d'une classe de maladie, nous proposons de comprendre l'extension du concept de maladie génétique à l'aune de cette interprétation mécaniste. Autrement dit, nous affirmons la possibilité d'esquisser les fondements d'une théorie génétique mécaniste de la maladie en général, qui échappe à toute accusation de génocentrisme tout en rendant compte de l'extension actuelle du concept de maladie génétique.

9. J.L. Casanova and L. Abel, «Human genetics of infectious diseases : a unified theory », European Molecular Biology Organization Journal, 2007, vol 26 (4), p. 915-922. 
Sébastien GROYER

Laboratoire de rattachement : PhiCo/NoSoPhi

Directrice de thèse : Catherine Larrère

sebgroyer@yahoo.fr

\section{Le libéralisme politique au secours du libéralisme économique}

«[...] dans le monde capitaliste comme dans le monde socialiste, on refuse de distinguer capitalisme et économie de marché ».

Fernand Braudel, La Dynamique du Capitalisme $e^{10}$

Dans la droite ligne de la citation de Fernand Braudel ci-dessus, il semble temps après la chute du communisme de tenter de séparer libéralisme économique, ou économie de marché, et capitalisme. Loin d'épuiser le libéralisme économique, le capitalisme peut être pensé comme une simple manifestation de ce libéralisme, encore inaboutie.

L'économie de marché se trouve ici repensée comme le système économique le plus capable de préserver la liberté individuelle, et, partant de la liberté, d'être relativement efficace par rapport aux autres systèmes plus dirigistes. Le lien entre liberté et efficacité est jugé par la prépondérance donnée à l'initiative individuelle, moteur de la création de richesses, que seul un système libéral fournit à un niveau optimal. Le capitalisme est de même redéfini comme le pouvoir au capital plutôt que comme la détention privée des moyens de production, qui englobe beaucoup de modes de gestion plus divers et peu assimilables au capitalisme réel. Le capitalisme est finalement un système économique relativement anti-libéral, sur une notion de liberté négative, l'absence d'oppression individuelle. 
$\mathrm{Si}$ le capitalisme est relativement antinomique avec le libéralisme économique, au nom d'une liberté non optimale, il peut être esquissé un système plus proche théoriquement de l'économie de marché, mais relativement différent du capitalisme.

En faisant appel aux travaux d'Albert Hirschmann sur la défense des intérêts des individus, qui réduit à deux solutions la capacité individuelle de défense, celle de partir ou de parler, ainsi qu'à Montesquieu et à l'opposition des pouvoirs, un autre système économique libéral peut être proposé. Ainsi, à l'oppression d'un groupe d'acteurs mus par leur intérêt propre est préféré l'équilibre des pouvoirs entre intérêts divergents, créateur d'une plus grande liberté individuelle. 
Eleni KonTOGIANNI

Laboratoire de rattachement : GRAMATA Thèse dirigée par : Michel Narcy elenikontogianni@hotmail.com

\section{Aristote, Heidegger, Bergson : substance et temporalité}

Dans Les Problèmes Fondamentaux de la Phénoménologie [329], Heidegger affirmait: "aucune tentative pour découvrir l'énigme du temps ne pourra se dispenser d'un débat avec Aristote ». Pour Heidegger, Aristote était le premier à porter au concept la compréhension naturelle du temps, et ceci de telle façon que la définition aristotélicienne «caractérise le temps dans la mesure où [...] nous devient accessible ce que nous appelons temps »[362].

En effet, dans la Physique IV, 11, en 219b2, Aristote définit le temps comme «le nombre du mouvement selon l'antérieur et postérieur». S'agissant d'une définition physique, le temps en tant que nombre se donne comme l'aspect quantifiable du mouvement, de telle façon qu'il ne peut être conçu qu'en tant qu'inséparable de celui-ci. Pourtant, cette définition du temps comme nombre met en relief deux conceptions du phénomène du temps : un temps qui correspond à une quantité temporelle donnée, en étant par là même observable et représentable, et un temps en devenir, en train de se réaliser suivant la réalisation du mouvement dont il est le nombre. Moyennant cette double perspective sur le temps, Aristote pose un dilemme : le mouvement assure au temps sa possibilité d'être, mais la réalité propre du temps comme nombre ne s'affirme-t-elle qu'après que celui-ci est conçu comme tel, comme nombre?

En clarifiant ainsi la problématique aristotélicienne, notre recherche porte sur la réception de ce questionnement respectivement par Heidegger et par Bergson. Dans son interprétation du traité aristotélicien, Heidegger, en 
mettant en perspective ses structures sous-jacentes, intègre la réflexion aristotélicienne dans sa structure de pensée propre. Cependant, donné sa critique de la durée bergsonienne résultant « d'une mauvaise intelligence de la compréhension aristotélicienne du temps » [328], Heidegger ne pourra jamais se concilier avec Bergson. Le problème de la manière d'être du temps est reçu selon deux perspectives différentes: en tant que temporalité du Dasein, en tant que durée de la conscience. 
Gauvain LECONTE

Laboratoire de rattachement: IHPST. Thèse dirigée par : Pierre Wagner gauvainleconte@wanadoo.fr

\section{Les deux racines du problème des prédictions scientifiques}

On considère souvent que les prédictions de nouveaux phénomènes comptent parmi les plus grands succès scientifiques et qu'une bonne théorie est une théorie dotée d'une forte capacité prédictive. Mais sait-on réellement comment nous réalisons des prédictions, et ce que l'on appelle la capacité prédictive d'une théorie ? Cette intervention vise à montrer comment ce problème spécifique a émergé récemment à partir de deux débats classiques - ceux concernant la théorie de la confirmation empirique des théories et le réalisme scientifique - et à présenter une des solutions à ce problème.

On peut distinguer les cas d'accommodation, où un phénomène est déjà connu avant la formulation d'une théorie qui l'explique (la théorie s'accommode aux faits) des cas de prédiction où le phénomène n'est découvert qu'après la théorie. L'exemple des prédictions réalisées en 1869 par Mendeleïev semble montrer que les phénomènes prédits par une théorie apportent plus de confirmation que les phénomènes accommodés et témoigner en faveur de la réalité des entités que suppose cette théorie.

Pourtant, si l'on veut soutenir ces thèses, il faut redéfinir la notion de prédiction scientifique et poser les problèmes suivants :

- Comment différencier prédiction et accommodation? Quels sont les différents types de prédictions et d'accommodations? C'est la question de la nature des prédictions.

- Les prédictions comptent-elles toujours plus que les accommodations? Ou ne comptent-elles que parfois plus, lorsqu'elles révèlent certaines qualités des théories qui les réalisent ? C'est le problème de la valeur des prédictions. 
Répondre à ces questions demande de décrire concrètement ce processus prédictif, et en s'inspirant de la manière dont on réalise des modèles statistiques prédictifs on peut définir non seulement la différence entre prédiction et accommodation mais aussi la notion de capacité prédictive d'un modèle comme étant sa capacité à équilibrer sa précision et sa simplicité.

La capacité prédictive d'un modèle résulte ainsi de sa capacité à équilibrer différentes exigences scientifiques: précision des données empiriques, simplicité des lois théoriques, unification de domaines expérimentaux variés, cohérence avec d'autres théories, etc. Même si les prédictions ne sont pas toujours supérieures aux accommodations, la confirmation d'un modèle sera donc proportionnelle à sa capacité prédictive. 
Sophie LEFEEZ

Laboratoire de rattachement : CETCOPRA Thèse dirigée par : Gérard Dubey, et financée par: EADS Innovation Works Sophie.Lefeez@malix.univ-paris1.fr

\section{La procédurisation dans les armées françaises}

La pratique guerrière, à l'instar d'autres pratiques, connaît actuellement en France une procédurisation. Dans la mesure où la pratique militaire fait cohabiter planification et adaptation, cet essor normatif (au sens large) interroge la place accordée à la flexibilité.

Depuis une quinzaine d'années, les textes de doctrine s'étendent de l'infanterie aux autres spécialités militaires. Cette réglementation vise non seulement à encadrer les pratiques, mais encore à les unifier pour des raisons financières et de commodité de travail en commun entre spécialités.

Dans cet environnement de plus en plus normatif, la déviance est de moins en moins acceptée par les acteurs eux-mêmes. Au contraire, tous les efforts sont faits pour tendre vers son respect, tant dans la conception des matériels que dans la formation ou l'emploi. Cela donne lieu à une standardisation des pratiques permettant une interchangeabilité des hommes et des matériels.

Si besoin, le milieu militaire va chercher dans la société civile une norme à laquelle il pourrait s'identifier et qui devient progressivement la référence à partir de laquelle le militaire doit justifier ses écarts. Ce faisant, certaines spécificités militaires ne sont plus perçues comme des attributs du métier de militaire mais comme des écarts à la norme, une déviance dont l'existence doit être justifiée - ou supprimée.

Par crainte de plaintes juridiques, des acteurs se réfugient dans un strict respect de la norme, au risque de perdre en flexibilité. Or, les normes ne sont valables que dans un cadre précis. En copiant des normes issues du civil, donc conçues dans et pour un temps de paix, la protection que l'on attend du 
strict respect de la norme peut se révéler illusoire : le moyen (la norme) est pris pour la fin (garantie de succès). En effet, comme la victoire passe par la mise en défaut de l'autre, la guerre menée n'est jamais celle préparée : elle déborde donc le cadre prédéfini d'applicabilité des normes. De fait, le travail des militaires, comme chez d'autres milieux professionnels ${ }^{11}$, consiste non pas à mettre en œuvre des procédures mais à gérer l'univers situationnel dans lequel se déroule l'action.

La prééminence croissante du geste technique transforme l'image du militaire et met en exergue la contradiction entre une pratique automatisée et l'expérience de la guerre comme expérience du chaos.

11. S. Poirot-Delpech, « Règles prescrites et règles auto-instituées dans le contrôle du trafic aérien », in La transgression des règles au travail, J. Girin et M. Grosjean (dir), Paris, L'Harmattan, 1996, p. 39-50. 
Tiffany PRINCEP

Laboratoire de rattachement : $\mathrm{PhiCo} / \mathrm{EXeCO}$ Thèse dirigée par : Jean-François Braunstein princeptiffany@hotmail.fr

\section{Qu'y a-t-il d'historique dans la sexualité ? Le cas de l'émergence du concept de perversion sexuelle au $\mathrm{XIX}^{\mathrm{e}}$ siècle}

En définissant la sexualité comme un champ polymorphe où savoirs, pouvoirs et plaisirs se sont historiquement croisés au cœur des sujets, Michel Foucault (1976) a ouvert un nouveau champ de recherche historique et proposé des outils féconds pour l'historicisation et par là la critique du sujet moderne.

Or le projet d'une histoire de la sexualité intervient chez Foucault au moment d'un remaniement méthodologique. Pour des raisons à la fois contextuelles et méthodologiques profondes qu'il s'est agi en premier lieu d'expliciter, l'analyse archéologique des discours sur le sexe a en effet été oblitérée par l'attention accrue portée à la dimension stratégique de ces discours, réflexion qui débouchera sur la méthode généalogique. Depuis L'archéologie du savoir (1969), les discours sont certes conçus comme des pratiques pourvues d'effets réels, mais dans La volonté de savoir (1976), il n'est quasiment plus question des «énoncés », qui constituaient le noyau de l'archéologie. En outre, les objets méthodologiques de la généalogie ne sont plus les discours, mais le «savoir/pouvoir», qui forme un niveau ontologico-historique propre.

Nous proposons donc en second lieu une critique des conséquences de cette méthodologie sur le projet d'une histoire de la sexualité, nous appuyant plus particulièrement sur le cas de l'émergence d'un discours psychopathologique à la fin du $\mathrm{XIX}^{\mathrm{e}}$ siècle. Parce qu'il évacue la question typiquement archéologique des conditions de possibilité de l'articulation 
réglée des objets, des concepts, et des choix théoriques au sein d'un discours, Foucault évacue la possibilité de décrire le processus par lequel la sexualité est positivement devenue un objet dont on peut parler - y compris pour en faire l'histoire. Ce n'est pas tant, en effet, le devenir objet de la sexualité qui occupe Foucault, mais le fait qu'elle soit historiquement devenue un objet à connaître.

Nous avançons enfin l'hypothèse de deux types de productions de la vérité sur le sexe à partir du milieu du $\mathrm{XIX}^{\mathrm{e}}$ siècle, certes stratégiquement cohérents, mais spécifiés au niveau archéologique. Constitution, d'une part, de la sexualité comme un domaine de vérité à découvrir et à fournir, nécessitant ce que l'on appellera une heuristique; constitution, d'autre part, de la sexualité en un domaine de signification à déchiffrer, appelant une herméneutique. Cette hypothèse permettra de relire à nouveaux frais l'émergence du concept de perversion sexuelle à la fin du XIX ${ }^{\mathrm{e}}$ siècle. 
David SIMONETTA

Laboratoire de rattachement : CHSPM Thèse dirigée par : André Charrak simonettadavid@yahoo.fr

\section{Empirisme ou phénoménologie : quel réductionnisme pour les contenus de conscience ?}

Malgré toutes les critiques qu'il a pu formuler ailleurs à l'encontre de l'empirisme, Husserl dans sa $14^{\mathrm{e}}$ leçon de Philosophie Première, rend un hommage appuyé à Locke. Il dit voir en lui l'initiateur d'une «authentique science intuitionniste de la conscience », parce qu'il aurait le premier, et mieux encore que Descartes, procédé à une description systématique des contenus de conscience. Cet hommage n'est pas isolé dans son œuvre. À la fin de sa longue critique du «naturalisme philosophique» dans $L a$ Philosophie comme science rigoureuse, Husserl explique que cette psychologie empiriste qu'il a tant combattue, peut néanmoins être considérée comme l'impulsion d'une tendance ayant conduit à la phénoménologie.

Comment comprendre que l'empirisme classique, dont Locke constitue la figure fondatrice, ait pu représenter aux yeux de Husserl à la fois l'ancêtre et l'adversaire de la phénoménologie ? L'objectif de cette intervention est de reconstruire l'argumentation de Husserl sous la forme d'un conflit entre deux sortes de réductionnismes en psychologie; eidétique d'une part, naturaliste d'autre part. Husserl rend hommage à Locke pour avoir, mieux que quiconque avant lui, compris l'importance du premier, et pour lui avoir donné une forme systématique, mais regrette qu'il n'ait pas pu mener jusqu'au bout son projet, et ce parce qu'il aurait finalement cherché à le rabattre sur le second type de réductionnisme.

Après avoir exposé en quoi consistent ces deux réductionnismes, puis reconstitué la critique de Husserl à l'égard de l'empirisme et montré les limites de son analyse, nous proposerons une clarification du sens et de 
l'usage du terme «intuition» chez les deux auteurs. Cette clarification terminologique permettra de mettre en évidence une divergence de fond entre les projets empiriste et phénoménologique. Cette divergence tient au rôle que l'on accorde au paradigme «naturaliste » en psychologie. Husserl rejette le naturalisme en ce qu'il implique fatalement, selon lui, une attitude réductionniste à l'égard des contenus de conscience. Mais Locke l'assumait pleinement parce qu'il le comprenait tout autrement: être naturaliste en matière de psychologie cela signifiait, pour Locke, intégrer la psychologie humaine à une échelle générale de l'intelligence, dont elle ne représente qu'un échelon, limité en perfection, et dont les limites peuvent être décrites. 
Sylvain THEULLE

Laboratoire de rattachement : PhiCo/NoSoPhi

Thèse dirigée par : Sandra Laugier

sylvain.theulle@wanadoo.fr

\section{La connaissance morale ordinaire}

L'approche théorique de la morale, du fait de son exigence d'unité et de simplicité, cherche à déduire l'ensemble des règles de conduite d'un concept fondamental (la raison, le plaisir, etc.), identifié au bien en soi. Or, le bien étant une notion abstraite, sans la moindre portée descriptive, cette identification du bien en soi à autre chose paraîtra toujours arbitraire, contestable. Ainsi, les théories morales sont victimes de leur adhésion à la thèse de la séparation des faits et des valeurs : un vocabulaire strictement évaluatif ne peut pas être appliqué sans arbitraire à la réalité, et n'est donc d'aucun secours pour formuler des règles de conduite adaptées aux situations.

Il convient donc de choisir une autre voie, qui prend pour point de départ les nombreux concepts éthiques appelés «épais » par Bernard Williams, qui ont une dimension descriptive. Le courage, le mensonge, la traîtrise sont des descriptions de caractères et d'actions, en même temps que l'évaluation morale de ceux-ci. Cette approche s'appuie sur le vocabulaire moral ordinaire. Elle est non théorique dans la mesure où rien n'impose que ces concepts doivent être logiquement articulés, ou réduits à une notion primitive. Et elle échappe à l'arbitraire, dans la mesure où le jugement moral découle directement des descriptions employant ces termes.

Cette seconde voie apparaît même comme la seule possible si l'on s'attache aux conditions de possibilité de l'apprentissage du discours moral. Les concepts éthiques épais sont les seuls qui peuvent être appris par l'enfant, car un concept éthique fin (tel que le bien), dépourvu de charge descriptive, est ipso facto dépourvu de conditions d'usage, et ne peut donc pas être appris. Il n'y a aucun moyen d'enseigner à un enfant ce que 
pourrait être une pure valeur. Même, les concepts épais ne pourraient être appris si l'enfant ne possédait pas une disposition immédiate à valoriser certains faits. Un enfant qui tiendrait son plaisir ou celui des autres pour un fait brut, axiologiquement neutre, serait définitivement hermétique aux notions évaluatives.

La nécessaire critique des conceptions morales ne prendra donc pas la forme d'un système sans présupposés, mais d'une discussion partant des conceptions morales communes, et qui vise à les amender, les supprimer, etc. 
Tonatiuh USECHE SANDOVAL

Directeur de thèse : Michel Bourdeau

Laboratoire de rattachement : PhiCo/NoSoPhi

tonatusco@yahoo.fr

\section{Quelle force militaire pour un Occident en paix ? La guerre de Crimée (1853-1856) interprétée par Auguste Comte}

Rares sont les publications qui ont étudié les propositions d'Auguste Comte pour réorganiser les forces armées d'un Occident sur la voie de l'état industriel. Ceci n'est pas étonnant. Selon les prévisions optimistes mais erronées de ce philosophe, l'exploitation pacifique des ressources naturelles devait devenir l'activité exclusive des sociétés occidentales. «La guerre sérieuse et durable doit totalement disparaitre de chez l'élite de chez l'Humanité », écrit-il en 1841. Comte pose une équation entre activités pacifiques et activités industrielles, terme qui englobe l'agriculture, la manufacture, le commerce et la banque. Cette équivalence l'a amené à établir une opposition radicale entre l'activité qui détruit et celle qui construit. Il semble que nous ayons affaire à une position absolument anti-militaire et utopique. Mais alors, comment comprendre que Comte ait donné son approbation en 1853 à l'intervention militaire en Crimée, lorsque la France et l'Angleterre se sont associées pour défendre la Turquie contre les tentatives d'expansion de la Russie ? Notre propos est de montrer que la thèse de Comte est pacifique, mais non pacifiste. Il ne nie pas l'importance provisoire des guerres dans l'essor de la sociabilité humaine. Il souligne même que l'activité militaire est plus synthétique que l'activité industrielle. Certes, Comte est un penseur du remplacement de la guerre par l'industrie. Seulement, ce remplacement n'est possible que s'il intervient de manière progressive. C'est pourquoi Comte s'est attaché à concevoir une transition et par suite une compatibilité entre ces deux activités pourtant opposées. La progressivité du changement fait que Comte s'interroge sur les formes de guerre et de forces armées compatibles avec l'instauration d'un système 
international où l'enrichissement des uns ne résulterait plus du dépouillement des autres. Nous commençons par montrer qu'un Occident industriel ne saurait pratiquer que des guerres défensives. Pour prévenir le retour des guerres de conquête, Comte propose de réduire le nombre de soldats, en confiant la défense nationale au patriotisme des citoyens, ensuite de remplacer les armées par des gendarmeries nationales, enfin de créer une gendarmerie occidentale dont la phase initiale de l'intervention militaire en Crimée offre la préfiguration. 
Fanny-Elisabeth RoLLET

Laboratoire de rattachement : PhiCo/NoSoPhi

Thèse dirigée par : Laurent Jaffro et Max Kistler

fannyeli.rollet@gmail.com

\section{L'agent et ses excuses en droit pénal : de l'intention criminelle aux dispositions coupables}

Notre projet est d'examiner les standards de la responsabilité et de l'irresponsabilité criminelle du point de vue de l'agentivité, à la lumière de concepts tirés de la philosophie de l'action (D. Davidson) et de théories de l'excuse formulées par des philosophes du droit anglo-saxons contemporains (H. L. A. Hart, G. Yaffe et R. Duff). On s'intéressera ici au problème de la caractérisation criminelle (i.e. au fait de savoir ce qui doit compter comme crime) sous l'angle du rapport qu'entretient cette qualification pénale avec les catégories de l'action imparfaite ou incomplète, et plus précisément avec la reconnaissance d'excuses dans le langage juridique.

On commencera par s'interroger sur la nature de l'excuse juridique au regard de la norme d'agentivité qu'elle sous-tend, pour se demander ensuite comment l'excuse juridique, dans ses mutations contemporaines, tend à être comprise en termes dispositionnels, alors même que certaines dispositions de l'agent revêtent une valeur juridique équivoque (aggravante et non atténuante de responsabilité).

Le problème de la nature de l'excuse et des limites de la criminalisation de l'intention peut ainsi conduire à une réflexion plus large propre à réinscrire l'ontologie pénale dans un cadre de philosophie politique: l'incrimination par des catégories pénales telles que celle de dangerosité (des dispositions ne valant plus que comme circonstances aggravantes) met en exergue la relation avec le modèle social et politique que cette ontologie est susceptible de promouvoir. 